\title{
Information acquisition and transparency in committees
}

\section{Journal Article}

\section{Author(s):}

Gersbach, Hans; Hahn, Volker

Publication date:

2012-05

Permanent link:

https://doi.org/10.3929/ethz-b-000048421

Rights / license:

In Copyright - Non-Commercial Use Permitted

\section{Originally published in:}

International Journal of Game Theory 41(2), https://doi.org/10.1007/s00182-011-0295-5 


\title{
Information acquisition and transparency in committees
}

\author{
Hans Gersbach • Volker Hahn
}

Accepted: 3 July 2011 / Published online: 24 July 2011

(C) Springer-Verlag 2011

\begin{abstract}
We study an intertemporal model of committee decision-making where members differ in their levels of efficiency. They may acquire costly information that enhances their ability to make a correct decision. We focus on the impact of transparency. We show that the principal's initial utility is higher under transparency, because members exert more effort, which makes correct decisions more likely. The principal also benefits from transparency later, unless transparency leads to an alignment of the signal qualities of highly efficient and less efficient committee members. In general, committee members are harmed by transparency. Together with the insights from the literature, our results may help to decide when transparency in committees is desirable.
\end{abstract}

Keywords Committees · Career concerns · Experts · Transparency · Information acquisition

JEL Classification D71

\section{Introduction}

Many important economic and political issues are decided by committees whose individuals have particular expertise. Company boards or monetary policy committees are examples in the economic realm. Parliamentary committees, the Council of Ministers in the European Union, or constitutional courts are examples in the political area.

H. Gersbach · V. Hahn ( $\varangle)$

CER-ETH, Center of Economic Research at ETH Zurich, Zürichbergstrasse 18, 8092 Zurich, Switzerland

e-mail: vhahn@ethz.ch

H. Gersbach

e-mail: hgersbach@ethz.ch 
Arguably, many of the decisions taken in these examples are complex, and members of the committee have to exert effort prior to meetings in order to thoroughly understand and evaluate the issues at hand. Hence the amount of information acquired by members is crucial for the quality of the committee's decision.

One recent phenomenon is that a variety of committee decision-making bodies are becoming more transparent by publishing deliberations and individual votes. ${ }^{1}$ In this paper we examine how transparency about the behavior of committee members impacts on the amount of information acquired by them and, accordingly, on the quality of decision-making in committees.

We analyze a two-period model. In each period, a committee of experts takes a decision whose quality affects the utility of a principal (such as the parliament, shareholders, ministers, etc.). The main ingredients of the model are that each committee member (he) has to exert effort to acquire information about the correctness of a decision and that members are heterogeneous in how effort translates into the likelihood of obtaining correct information. The principal (she) cannot observe committee members' types directly. To improve committee decision-making, the principal would like members to exert high effort. Moreover, she aims at improving the composition of the committee over time.

Committee members are motivated by career concerns. It is plausible that members are concerned about their prestige and the satisfaction gained by working on the committee and thus, in turn, about their re-appointment prospects. The desire to be re-appointed creates incentives to acquire information, as the principal will tend to re-appoint only those members who display higher quality in decision-making than the average in the pool of potential experts.

The principal has two means of interfering with the workings of the committee. First, she can choose either opaque or transparent voting at the beginning of the game. When the principal opts for transparency, she can evaluate the quality of a member by observing the individual's decisions. When the principal decides to have an opaque committee, she observes the collective decision alone. Second, after the committee has made its decision in the first period, she can re-appoint members or replace them by new experts.

Our main insights are as follows. First, we show that under transparency the principal is always better off in the first period, because transparency induces higher effort on behalf of committee members and thus improves decision-making in the first period.

Second, the principal also benefits from transparency in the second period, unless the higher effort levels induced by transparency lead to a strong alignment of the signal qualities of highly efficient and less efficient members. Under transparency, the principal can observe individual votes, which, for given effort levels, makes it easier to identify the individual competency of members. If, however, the increases in effort levels induced by transparency lead to a strong convergence of the qualities of members' signals, then it becomes difficult to distinguish between highly efficient members and less efficient ones. We identify a critical property that guarantees that the principal

\footnotetext{
${ }^{1}$ Examples are the Federal Open Market Committee, the U.S. Supreme Court, or the Monetary Policy Committee of the Bank of England.
} 
benefits from transparency in the second period. We also provide a counterexample in which the principal's second-period utility is higher under opacity.

Third, we generalize our findings to the case where experts do not know their own abilities. In this set-up, transparency is superior to opacity for the principal under all circumstances.

Fourth, we show that transparency makes committee members worse off, because they have to invest more in effort and their re-appointment chances are lower. This might explain a certain initial reluctance on the part of various committees to make their decision-making transparent. For example, the FOMC did not adopt transparent decision-making voluntarily.

Fifth, we explore how the first and second insight are influenced by several factors. In particular, we consider alternative assumptions on how effort translates into the quality of an individual's signal, pre-meetings, committee size, and the case where the principal has no information regarding the quality of the decision. Overall, the beneficial effects of transparency survive these extensions.

In Sect. 9, taking the findings of this paper and the existing literature into account, we will draw a more general conclusion. We elaborate on the circumstances under which transparency is beneficial to committee designers and those under which it is not.

Our paper combines two strands of literature. The first is concerned with the impact of transparency requirements on the quality of committee decision-making if the abilities of members are exogenously given. ${ }^{2}$ This literature has shown that transparency may distort the behavior of individual committee members, because they are not only interested in the impact of their vote on the outcome of decision-making but attempt to use their vote as a signal of a particular type. This effect is taken into account in Fingleton and Raith (2005), Gersbach and Hahn (2008), Levy (2007), Sibert (2003), and Stasavage (2007). ${ }^{3}$ In our model, for a given signal of a member, the vote that maximizes a member's reputation also maximizes the principal's utility. Thus the distortionary effect of transparency studied in these papers does not occur. Additional effects have been identified in the literature on transparency and committee decision-making. Sibert (2003) analyzes the impact of transparent decision-making on reputation-building if central bankers may have an incentive to increase output above the natural rate. Levy (2007) shows that opaque decision-making induces members to comply with pre-existing biases, as the signaling incentives in such cases are not eliminated. The disadvantage of secrecy can be obviated if appropriate voting rules are used. Gersbach and Hahn (2008) identify an intertemporal trade-off between transparency and opacity. While secrecy initially makes for better decisions because uninformed committee members have no incentive to mimic informed members, it hampers the principal's ability to dismiss manifestly less well-informed members.

\footnotetext{
${ }^{2}$ Holmström (1982) is a seminal contribution to the literature on experts motivated by career concerns. Other important contributions include Scharfstein and Stein (1990), Trueman (1994), and Ottaviani and Sørensen (2001).

3 Visser and Swank (2007) present another interesting model where experts derive utility from being perceived as competent. Prat (2005) examines transparency for a career concern model with one individual expert.
} 
The second strand of literature is concerned with information acquisition and voting in committees. ${ }^{4}$ Gersbach (1995) studies agents' incentives to acquire information if decisions are made by majority rule. He characterizes situations where the committee acts as if it were fully informed and where this is preferred to ignorance by a majority of members or the whole committee. Mukhopadhaya (2003) and Persico (2004) examine the role of committee size for incentives to acquire information. Both find that large committees lead to a severe underprovision of information. ${ }^{5}$ Gerardi and Yariv (2008) study the optimal committee size and decision rule. We examine here how the incentives to acquire information interact with the transparency regime when members have career concerns.

This paper is organized as follows: In Sect. 2 we outline our model. We analyze the equilibrium in the second period for transparency and opacity in Sect. 3. Subsequently, in Sects. 4 and 5, we derive the equilibria under transparency and opacity. We compare the utility under transparency and under opacity for the principal and the committee members in Sect. 6. In Sect. 7, we analyze our model for the alternative assumption that experts do not know their own abilities. The robustness of our findings and additional extensions to our model are discussed in Sect. 8. Section 9 concludes.

\section{Model}

We consider a two-period model of a committee comprising $N$ experts $(N>1)$. For simplicity of exposition we assume that $N$ is odd. Committee members make a decision $d_{t} \in\{-1,+1\}$ by majority rule in each period $t=1,2$. We use $d_{t}^{i} \in\{-1,+1\}$ to denote the individual vote of member $i(i=1, \ldots, N)$.

Committee members are appointed by a principal who benefits if the committee reaches a decision that corresponds to the state of the world, which is given by $s_{t} \in\{-1,+1\}$. The principal has the following utility function in each period $t=1,2$ :

$$
u_{t}^{P}= \begin{cases}1 & \text { if } d_{t}=s_{t} \\ 0 & \text { if } d_{t} \neq s_{t}\end{cases}
$$

Without loss of generality, we have normalized the principal's benefits from a correct decision to 1 . We assume that nature chooses $s_{t}=-1$ and $s_{t}=1$ with equal probability. The state of the world is unknown to the principal at the beginning of period $t$. However, it becomes common knowledge after the committee has made its decision. There is a common discount factor $\delta$ for the principal and experts.

There are two types of experts, highly efficient experts and less efficient experts. We assume that the level of efficiency is private information. The prior probability of an expert being highly efficient amounts to $q^{H}\left(0<q^{H}<1\right)$. The probability for the less efficient type is denoted by $q^{L}=1-q^{H}$. Each expert $i$ may invest effort $e_{t}^{i} \in[0, E](E>0)$ into information acquisition about the state of the world.

\footnotetext{
4 For a survey of this literature see Gerling et al. (2005).

5 As a member benefits from information acquisition by others, this is a classical team-work problem of the kind discussed by Marschak and Radner (1972).
} 
The effort of experts is unobservable to the principal. Each expert $i$ then receives a signal $\sigma_{t}^{i} \in\{-1,+1\}$ about the state of the world. The quality of the signal depends on the expert's type and on the effort exerted by him. For highly efficient experts, the signal is correct with probability $p^{H}\left(e_{t}^{i}\right)$; with probability $1-p^{H}\left(e_{t}^{i}\right)$ the signal is incorrect. A less efficient expert has a probability $p^{L}\left(e_{t}^{i}\right)$ of receiving a correct signal. Thus the effort in period $t$ only affects the quality of the signal in the same period. The probability of an individual expert receiving a correct signal is independent of whether other experts have received correct or incorrect signals.

We make the following assumptions about $\left.\left.p^{H}:[0, E] \rightarrow\right] 1 / 2,1\right]$ and $p^{L}$ : $[0, E] \rightarrow] 1 / 2,1]:$

$$
\begin{array}{ccr}
p^{H}\left(e_{t}^{i}\right)>p^{L}\left(e_{t}^{i}\right) & \frac{\partial p^{H}}{\partial e_{t}^{i}}\left(e_{t}^{i}\right) \geq \frac{\partial p^{L}}{\partial e_{t}^{i}}\left(e_{t}^{i}\right) & \forall e_{t}^{i} \in[0, E[, \\
\frac{\partial p^{X}}{\partial e_{t}^{i}}\left(e_{t}^{i}\right)>0 & \frac{\partial^{2} p^{X}}{\partial\left(e_{t}^{i}\right)^{2}}\left(e_{t}^{i}\right)<0 & \forall e_{t}^{i} \in[0, E[, \forall X \in\{H, L\}, \\
\lim _{e_{t}^{i} \rightarrow E} \frac{\partial p^{X}}{\partial e_{t}^{i}}\left(e_{t}^{i}\right)<\frac{1}{\delta B} & \lim _{e_{t}^{i} \rightarrow 0} \frac{\partial p^{X}}{\partial e_{t}^{i}}\left(e_{t}^{i}\right)=\infty & \forall X \in\{H, L\},
\end{array}
$$

where parameter $B>0$ will be explained below.

Hence we assume that experts with high efficiency have a higher probability of obtaining a correct signal for a given level of effort. Moreover, a marginal increase in effort results in a weakly higher increase in the precision of the signal for highly efficient members over and against less efficient members. The assumptions on $\lim _{e_{t}^{i} \rightarrow E} \frac{\partial p^{X}}{\partial e_{t}^{i}}\left(e_{t}^{i}\right)$ and $\lim _{e_{t}^{i} \rightarrow 0} \frac{\partial p^{X}}{\partial e_{t}^{i}}\left(e_{t}^{i}\right)$ are made for analytical convenience and guarantee the existence of interior solutions.

We assume that experts obtain utility from being on the committee. These benefits amount to $B>0$ in each period. Benefits $B$ may comprise wages. They may also be affected by the prestige and satisfaction involved in working on the committee. Moreover, experts incur costs from exerting effort. If they do not hold office, utility is normalized to 0 . The utility of an expert in period $t$ is given by

$$
u_{t}^{i}= \begin{cases}B-e_{t}^{i} & \text { if the expert is on the committee } \\ 0 & \text { if the expert is not on the committee }\end{cases}
$$

We assume $B>E$, i.e. an expert prefers to be a committee member even if he exerts the maximum level of effort $E$.

The sequence of events is as follows:

\section{Period 0}

- The principal decides on the transparency regime.

\section{Period 1}

- The principal appoints committee members from a pool of candidates.

- Nature chooses the state of the world.

- Experts may invest in information acquisition.

- Experts receive private signals about the state of the world.

- Experts vote simultaneously. 
- Under transparency, individual voting records $\left(d_{1}^{i}\right)_{i=1}^{N}$ are published. Under opacity, only the decision $d_{1}$ becomes known.

\section{Period 2}

- The principal observes $s_{1}$ and may either re-appoint each committee member or replace him by a new member from a pool of candidates.

- The remaining events are identical to those described for period $1 .^{6}$

We will identify perfect Bayesian Nash equilibria of this game. Thus each member's effort levels and votes must be optimal, given the principal's re-appointment scheme and the other members' decisions. Moreover, the principal's re-appointment scheme must be optimal, given the behaviors of committee members. We introduce several plausible equilibrium refinements. Later we will show that perfect Bayesian Nash equilibria satisfying these refinements actually exist.

First, we introduce the following tie-breaking rule: experts vote in line with their signals if they are indifferent between votes. For example, this refinement rules out implausible equilibria under opacity where all members always vote for the same decision irrespective of their signal. No profitable deviation would exist in this case, as all experts would never be pivotal.

Second, we make the assumption that re-appointment probabilities are symmetric in the following sense. Under transparency, the probability of re-appointment depends only on the correctness of the individual vote, where we call an individual vote correct if $d_{1}^{i}=s_{1}$. Under opacity, the re-appointment probability depends only on the correctness of the committee decision, i.e. on whether $d_{1}=s_{1}$ holds or not. Thus we use $\mu_{T}^{i}\left(Z^{i}\right)\left(Z^{i} \in\{C, W\}\right)$ to denote the probability of an expert being re-appointed under transparency if he has made a correct $\left(Z^{i}=C\right)$ or a wrong $\left(Z^{i}=W\right)$ decision $\left(\mu_{T}^{i}\left(Z^{i}\right) \in[0,1]\right){ }^{7}$ Similarly, we introduce $\mu_{O}^{i}(Z)(Z \in\{C, W\})$ to denote the re-appointment probability of expert $i$ if the committee has made the correct $(C)$ or the wrong $(W)$ decision.

Third, we assume that the re-appointment scheme does not punish members for correct individual votes under transparency and for correct committee decisions under opacity. Formally, this refinement can be stated as

$$
\begin{aligned}
& \mu_{T}^{i}(C) \geq \mu_{T}^{i}(W) \quad \forall i \in\{1,2, \ldots, N\} \\
& \mu_{O}^{i}(C) \geq \mu_{O}^{i}(W) \quad \forall i \in\{1,2, \ldots, N\}
\end{aligned}
$$

It excludes implausible equilibria where experts deliberately make wrong decisions in order to signal high ability.

\footnotetext{
6 We assume that the states of the world are stochastically independent across periods.

7 In principle, a member's probability of re-appointment could also depend on the other members' votes. Such behavior could be optimal to the principal only if she were indifferent between dismissing and re-appointing the respective member. It can be shown that our implicit assumption that other members' votes do not affect a member's re-appointment probability does not affect our findings.
} 


\section{Behavior of experts in the second period}

The behavior of experts in the second period does not depend on the transparency regime. It is obvious that experts will never exert effort in the second period, i.e. $e_{2}^{H}=e_{2}^{L}=0$ holds. Thus the utility of an expert holding office in the second period amounts to $u_{2}^{i}=B$. We summarize this finding in the following lemma:

Lemma 1 Under transparency and opacity, experts choose effort levels $e_{2}^{i}=0$ in the second period.

It will be useful to introduce $\mathcal{P}\left(p^{1}, p^{2}, \ldots, p^{N}\right)$ as the probability of the committee reaching a correct decision if each individual member $i \in\{1, \ldots, N\}$ makes a correct decision with probability $p^{i}$ and if $p^{i}$ is independent of whether other experts make a correct decision or not. ${ }^{8}$ Importantly, $\mathcal{P}\left(p^{1}, p^{2}, \ldots, p^{N}\right)$ is strictly increasing in all of its arguments for $\left.p^{i} \in\right] 0,1[\forall i \in\{1, \ldots, N\}$, as each member is pivotal with strictly positive probability for $\left.p^{i} \in\right] 0,1[\forall i \in\{1, \ldots, N\}$.

According to the tie-breaking rule introduced in Sect. 2, experts always vote in line with their private signals in the second period. This implies that the probability of a highly efficient expert voting correctly amounts to $p^{H}(0)$. The respective probability for a less efficient expert amounts to $p^{L}(0)$. Recall that our assumptions about $p^{X}\left(e_{t}^{i}\right)$ imply that the probability of an expert choosing the correct vote is strictly higher in the case of a highly efficient expert compared to the case of a less efficient expert for a specified level of effort. Because $\mathcal{P}\left(p^{1}, p^{2}, \ldots, p^{N}\right)$ is strictly increasing with each $p^{i}$, we therefore obtain:

Lemma 2 Under transparency and opacity, the principal's optimal re-appointment decision is ${ }^{9}$

member $i$ is $\left\{\begin{array}{l}\text { re-appointed } \text { if } i \text { is highly efficient with a probability larger than } q^{H} \\ \text { dismissed }\end{array}\right.$

The respective probabilities of a member being highly efficient are determined by Bayesian updating. They are calculated for both scenarios in the following.

\section{Transparency}

We begin our analysis of transparent committees by examining members' optimal voting behavior in the first period. Assume a committee member of type $X \in\{L, H\}$ has exerted effort $e_{1}^{i}$ and has received signal $\sigma_{1}^{i}$. This implies that $s_{1}=\sigma_{1}^{i}$ is correct with probability $p^{X}\left(e_{1}^{i}\right)>1 / 2$. There are two cases in line with our assumptions

\footnotetext{
8 Of course, members' signals are positively correlated. However, the probability of a member voting correctly is independent of the correctness of other members' votes because the probability of a member receiving a correct signal is independent of whether other members have received a correct signal.

${ }^{9}$ If the probability of $i$ being highly efficient amounts to $q^{H}$, the government is indifferent between dismissal and re-appointment.
} 
about re-appointment schemes. First, the probability of re-appointment may be insensitive to the correctness of the individual vote $\left(\mu_{T}^{i}(C)=\mu_{T}^{i}(W)\right)$. According to our tie-breaking rule, the committee member will make the decision suggested by his signal. Second, the probability of re-appointment may be sensitive to the correctness of the individual vote, i.e. $\mu_{T}^{i}(C)>\mu_{T}^{i}(W)$ holds. If the committee member votes for $\sigma_{1}^{i}$, the probability of being re-appointed amounts to $p^{X}\left(e_{1}^{i}\right) \mu_{T}^{i}(C)+$ $\left(1-p^{X}\left(e_{1}^{i}\right)\right) \mu_{T}^{i}(W)$. If the expert votes for $-\sigma_{1}^{i}$, then the probability of re-appointment is given by $\left(1-p^{X}\left(e_{1}^{i}\right)\right) \mu_{T}^{i}(C)+p^{X}\left(e_{1}^{i}\right) \mu_{T}^{i}(W)$. Because the expert tries to maximize his re-appointment chances, it is strictly optimal to vote for $\sigma_{1}^{i}$ if

$$
p^{X}\left(e_{1}^{i}\right) \mu_{T}^{i}(C)+\left(1-p^{X}\left(e_{1}^{i}\right)\right) \mu_{T}^{i}(W)>\left(1-p^{X}\left(e_{1}^{i}\right)\right) \mu_{T}^{i}(C)+p^{X}\left(e_{1}^{i}\right) \mu_{T}^{i}(W)
$$

This inequality always holds for $p^{X}\left(e_{1}^{i}\right)>1 / 2$ and $\mu_{T}^{i}(C)>\mu_{T}^{i}(W)$. Hence it is always optimal to vote in line with one's signal. We summarize this finding in the following lemma:

Lemma 3 Under transparency, experts always make the decision suggested by their signals in the first period.

Next, we analyze the optimal choice of effort in the first period. An expert $i$ with efficiency level $X \in\{H, L\}$ chooses $e_{1}^{i}$ in order to maximize

$$
B-e_{1}^{i}+\delta\left[p^{X}\left(e_{1}^{i}\right) \mu_{T}^{i}(C)+\left(1-p^{X}\left(e_{1}^{i}\right)\right) \mu_{T}^{i}(W)\right] B
$$

This yields the first-order condition ${ }^{10}$

$$
1=\delta\left[\mu_{T}^{i}(C)-\mu_{T}^{i}(W)\right]\left(p^{X}\right)^{\prime}\left(e_{1}^{i}\right) B
$$

As a consequence, the optimal level of effort under transparency is given by

$$
e_{1, T}^{i, X}= \begin{cases}\left(\left(p^{X}\right)^{\prime}\right)^{-1}\left(\frac{1}{\left(\mu_{T}^{i}(C)-\mu_{T}^{i}(W)\right) \delta B}\right) & \text { for } \mu_{T}^{i}(C)>\mu_{T}^{i}(W) \\ 0 & \text { for } \mu_{T}^{i}(C)=\mu_{T}^{i}(W) .\end{cases}
$$

Equation (8) and our assumptions about $p^{X}$ immediately imply the following lemma:

Lemma 4 Under transparency, every expert $i$ chooses a weakly higher level of effort if he is highly efficient rather than less efficient. The probability of expert $i$ choosing a correct vote in the first period is always strictly higher if he is highly efficient rather than less efficient.

\footnotetext{
10 Our assumption $p^{X^{\prime \prime}}\left(e_{t}^{i}\right)<0$ implies that the solution to the first-order condition always represents a maximum.
} 
In the following, we turn to the optimal re-appointment scheme under transparency. According to Lemma 2, we have to determine the probability that an expert who has voted correctly is highly efficient. Bayes' rule implies

$$
\kappa^{i, H}=\frac{q^{H} p^{H}\left(e_{1, T}^{i, H}\right)}{q^{H} p^{H}\left(e_{1, T}^{i, H}\right)+q^{L} p^{L}\left(e_{1, T}^{i, L}\right)},
$$

where we have used the fact that the probability of expert $i$ being highly efficient and choosing the correct vote is $q^{H} p^{H}\left(e_{1, T}^{i, H}\right)$. The probability of a less efficient committee member receiving a correct signal is $q^{L} p^{L}\left(e_{1, T}^{i, L}\right)$. Probability $\kappa^{i, H}$ is higher than the probability of a newly appointed candidate being highly efficient if

$$
\frac{q^{H} p^{H}\left(e_{1, T}^{i, H}\right)}{q^{H} p^{H}\left(e_{1, T}^{i, H}\right)+q^{L} p^{L}\left(e_{1, T}^{i, L}\right)}>q^{H},
$$

which is equivalent to

$$
p^{H}\left(e_{1, T}^{i, H}\right)>q^{H} p^{H}\left(e_{1, T}^{i, H}\right)+\left(1-q^{H}\right) p^{L}\left(e_{1, T}^{i, L}\right) .
$$

This inequality always holds, because $0<q^{H}<1$ and $p^{H}\left(e_{1, T}^{i, H}\right)>p^{L}\left(e_{1, T}^{i, L}\right)$, which follows from Lemma 4 . By virtue of Lemma 2 , it is thus strictly optimal to re-appoint a committee member who has voted correctly. Analogous arguments show that it strictly optimal to dismiss a committee member who has chosen the wrong decision. We summarize these results in the following lemma:

Lemma 5 Under transparency, the optimal re-appointment scheme is given by

$$
\mu_{T}^{i}\left(Z^{i}\right)= \begin{cases}1 & \text { for } Z^{i}=C \\ 0 & \text { for } Z^{i}=W\end{cases}
$$

Using (8), the equilibrium level of effort under transparency is given by ${ }^{11}$

$$
e_{1, T}^{X}=\left(\left(p^{X}\right)^{\prime}\right)^{-1}\left(\frac{1}{\delta B}\right) \quad \forall X \in\{H, L\} .
$$

\section{Opacity}

It is obvious that under opacity experts will always vote in line with their signals in the first period. This will increase their chances of re-election for $\mu_{O}^{i}(C)>\mu_{O}^{i}(W) .{ }^{12}$

\footnotetext{
11 As the level of effort is identical for all members of a particular type $X$, we do not use an additional index $i$ here.

12 Each individual member knows that his vote will only affect the outcome if there is a draw among the other members. In this case, both choices are equally likely and it is optimal to vote in line with one's signal.
} 
For $\mu_{O}^{i}(C)=\mu_{O}^{i}(W)$ this behavior is chosen in accordance with our tie-breaking rule. What remains to be examined is the level of effort chosen by highly efficient and less efficient experts.

For this purpose, it will be useful to introduce the set of combinations of the other experts' votes for which $i$ is pivotal $\mathbb{C}_{i}^{p i v}:=\left\{\left(c_{j}\right)_{j \neq i} \in\{0,1\}^{N-1} \mid \sum_{j \neq i} c_{j}=(N-1) / 2\right\}$, where we use the convention that $c_{j}=1$ stands for a correct vote of expert $j$ and $c_{j}=0$ for an incorrect vote. Moreover, the set of combinations of the other experts' votes for which a correct decision is taken irrespective of $i$ 's vote is $\mathbb{C}_{i}^{c o r}:=$ $\left\{\left(c_{j}\right)_{j \neq i} \in\{0,1\}^{N-1} \mid \sum_{j \neq i} c_{j} \geq(N+1) / 2\right\}$. With these sets we can express the probability of the expert being pivotal as

$$
\begin{aligned}
\chi^{i}=\sum_{\left(c_{j}\right)_{j \neq i} \in \mathbb{C}_{i}^{p i v}} \prod_{j \in\{1, \ldots, N\} \backslash\{i\}} & \left(q^{H} p^{H}\left(e_{1}^{j, H}\right)+q^{L} p^{L}\left(e_{1}^{j, L}\right)\right)^{c_{j}} \\
& \times\left(1-q^{H} p^{H}\left(e_{1}^{j, H}\right)-q^{L} p^{L}\left(e_{1}^{j, L}\right)\right)^{1-c_{j}}
\end{aligned}
$$

and the probability of a correct decision being taken irrespective of $i$ 's vote as

$$
\begin{aligned}
\psi^{i}=\sum_{\left(c_{j}\right)} \prod_{j \neq i \in \mathbb{C}_{i}^{c o r}} \prod_{j \in\{1, \ldots, N\} \backslash\{i\}} & \left(q^{H} p^{H}\left(e_{1}^{j, H}\right)+q^{L} p^{L}\left(e_{1}^{j, L}\right)\right)^{c_{j}} \\
& \times\left(1-q^{H} p^{H}\left(e_{1}^{j, H}\right)-q^{L} p^{L}\left(e_{1}^{j, L}\right)\right)^{1-c_{j}} .
\end{aligned}
$$

The effort level $e_{1}^{i, X}$ of expert $i$ with efficiency level $X$ is chosen so as to maximize $i$ 's expected level of utility, which is

$$
\begin{aligned}
\phi^{i, X}:= & B-e_{1}^{i, X} \\
& +\delta\left[\left(\psi^{i}+\chi^{i} p^{X}\left(e_{1}^{i, X}\right)\right) \mu_{O}^{i}(C)+\left(1-\psi^{i}-\chi^{i} p^{X}\left(e_{1}^{i, X}\right)\right) \mu_{O}^{i}(W)\right] B,
\end{aligned}
$$

where we have utilized our previous result that expert $i$ will not exert effort in the second period $\left(e_{2, O}^{i, X}=0, \forall X \in\{H, L\}\right)$. Optimization yields the first-order condition

$$
1=\delta \chi^{i} \cdot\left(p^{X}\right)^{\prime}\left(e_{1}^{i, X}\right)\left[\mu_{O}^{i}(C)-\mu_{O}^{i}(W)\right] B .
$$

Thus the optimal level of effort under opacity is

$$
e_{1, O}^{i, X}= \begin{cases}\left(\left(p^{X}\right)^{\prime}\right)^{-1}\left(\frac{1}{\left(\mu_{O}^{i}(C)-\mu_{O}^{i}(W)\right) \chi^{i} \delta B}\right) & \text { for } \chi^{i}\left(\mu_{O}^{i}(C)-\mu_{O}^{i}(W)\right)>0 \\ 0 & \text { for } \chi^{i}\left(\mu_{O}^{i}(C)-\mu_{O}^{i}(W)\right)=0\end{cases}
$$

Our assumptions about $p^{X}($.$) again imply:$ 
Lemma 6 Under opacity, each expert $i$ chooses a weakly higher level of effort if he is highly efficient rather than less efficient. His probability of obtaining a correct signal is strictly higher if he is highly efficient rather than less efficient.

Because in the first period highly efficient experts are always more likely to vote correctly compared to less efficient committee members, a committee that has made a correct decision is always strictly more likely to arrive at a correct decision in the second period than a newly appointed committee. Similarly a committee that has made a wrong decision is always strictly less likely to arrive at a correct decision in the second period than a newly appointed committee. These findings imply the next lemma, which is shown formally in Appendix A:

Lemma 7 Under opacity, the principal's optimal re-appointment scheme is given by

$$
\mu_{O}^{i}(Z)= \begin{cases}1 & \text { for } Z=C \\ 0 & \text { for } Z=W\end{cases}
$$

Now the member $i$ 's level of effort in an equilibrium under opacity can be written as

$$
e_{1, O}^{i, X}=\left(\left(p^{X}\right)^{\prime}\right)^{-1}\left(\frac{1}{\chi^{i} \delta B}\right) .
$$

Condition (20) holds for all committee members $i$ and $\chi^{i}$ depends on the effort levels of all other members. In Appendix B we show

Lemma 8 The system of $2 N$ equations represented by (20) for $i \in\{1, \ldots, N\}$ and $X \in\{H, L\}$ always has a solution.

While the solution is not unique in general, all of our findings will hold independently of which equilibrium is chosen.

\section{Comparison of transparency and opacity}

\subsection{The first period}

Finally, we compare the principal's utility under transparency and under opacity. Under transparency, all highly efficient experts will choose identical effort levels $e_{1, T}^{H}$ in the first period. Their vote is correct with probability $p^{H}\left(e_{1, T}^{H}\right)$. A less efficient expert's likelihood of choosing the correct vote is $p^{L}\left(e_{1, T}^{L}\right)$. Because the prior probability of a member being highly efficient is $q^{H}$, the probability of an expert making a correct decision in the first period is given by

$$
\rho_{1, T}:=q^{H} p^{H}\left(e_{1, T}^{H}\right)+\left(1-q^{H}\right) p^{L}\left(e_{1, T}^{L}\right) .
$$

Hence the principal's expected utility in the first period is

$$
u_{1, T}^{P}=\mathcal{P}\left(\rho_{1, T}, \rho_{1, T}, \ldots, \rho_{1, T}\right) .
$$


Under opacity, the probability of an individual expert $i \in\{1, \ldots, N\}$ voting correctly in the first period is given by

$$
\rho_{1, O}^{i}:=q^{H} p^{H}\left(e_{1, O}^{i, H}\right)+\left(1-q^{H}\right) p^{L}\left(e_{1, O}^{i, L}\right) .
$$

Notice that $\rho_{1, O}^{i}$ may be different across experts, whereas $\rho_{1, T}$ is always identical for all experts. This is a consequence of the fact that under opacity asymmetric equilibria may exist where different members with the same level of efficiency choose different effort levels.

With the help of $\rho_{1, O}^{i}$, the principal's expected utility under opacity can be stated as

$$
u_{1, O}^{P}=\mathcal{P}\left(\rho_{1, O}^{1}, \rho_{1, O}^{2}, \ldots, \rho_{1, O}^{N}\right)
$$

Because of $\chi^{i}<1$, equations (13) and (20) yield $e_{1, T}^{X}>e_{1, O}^{i, X} \forall i \in\{1, \ldots, N\}$. Together with the observation that $p^{X}($.) is a strictly monotonically increasing function this implies $\rho_{1, T}>\rho_{1, O}^{i} \forall i \in\{1, \ldots, N\}$. Intuitively, transparency leads to higher effort levels of all members, irrespective of the equilibrium chosen under opacity. Thus each member votes for the correct option with higher probability than under opacity. As mentioned above, probability $\mathcal{P}\left(p^{1}, p^{2}, \ldots, p^{N}\right)$ is strictly monotonically increasing in all of its arguments. This yields the following proposition:

Proposition 1 The principal's first-period utility is always higher under transparency than under opacity, i.e. $u_{1, T}^{P}>u_{1, O}^{P}$.

\subsection{The second period}

In the second period, it appears plausible that the principal's utility will be higher under transparency. This is suggested by the observation that transparency may improve the principal's ability to distinguish highly efficient from somewhat less efficient committee members. However, this is not always the case, as transparency might cause a convergence of the signal qualities for highly efficient and less efficient members. This countervailing effect may reduce the principal's ability to distinguish between highly efficient and less efficient members.

In order to make this more precise, we need to introduce additional notation. Suppose that experts could be rewarded for making correct decisions in a particular period. We use $r \in[0, \delta B]$ to denote this reward in utility units. Moreover, we introduce $e^{H}(r)=\left(\left(p^{H}\right)^{\prime}\right)^{-1}\left(\frac{1}{r}\right)$ and $e^{L}(r)=\left(\left(p^{L}\right)^{\prime}\right)^{-1}\left(\frac{1}{r}\right)$ to denote the optimal choice of high and low ability types respectively. Now we are in a position to define the following property:

Definition 1 The functions $p^{H}(e)$ and $p^{L}(e)$ have the property of non-decreasing quality differences (NDQD) if the following condition holds for all $r \in[0, \delta B]$ :

$$
\frac{d\left(p^{H}\left(e^{H}(r)\right)-p^{L}\left(e^{L}(r)\right)\right)}{d r} \geq 0 .
$$


Intuitively, this property ensures that a marginal increase in the rewards for correct decisions will increase the signal quality gap between highly efficient and less efficient members.

Condition (25) can be rewritten as $\frac{d p^{H}\left(e^{H}(r)\right)}{d r} \geq \frac{d p^{L}\left(e^{L}(r)\right)}{d r}$. NDQD is stronger than our assumption $p^{H^{\prime}}(e) \geq p^{L^{\prime}}(e) \forall e \in\left[0, E\left[\right.\right.$, because of $e^{H}(r) \geq e^{L}(r)$ and the concavity of $p^{H}(e)$.

NDQD is fulfilled, for example, if $p^{H}(e)=p^{L}(e)+c(c>0)$ holds in addition to our assumptions. This is a consequence of the fact that for this specification $e^{H}(r)=e^{L}(r)$ holds for all $r \in[0, \delta B]{ }^{13}$

In Appendix $\mathrm{C}$ we show

Proposition 2 If the functions $p^{H}(e)$ and $p^{L}(e)$ satisfy $N D Q D$, then the principal's second-period utility is always higher under transparency than under opacity, i.e. $u_{2, T}^{P}>u_{2, O}^{P}$.

If NDQD does not hold, then it is conceivable that the principal's utility in the second period is higher under opacity. Under transparency, the increased effort might lead to almost identical signal qualities in the first period, which makes it virtually impossible for the principal to distinguish highly efficient from less efficient experts.

In the next proposition, we show that this is indeed possible.

Proposition 3 If NDQD does not hold, then the principal's utility in the second period may be higher under opacity.

Proposition 3 is proved by an example, which is given in Appendix D. The basic idea underlying the example is that both functions $p^{H}(e)$ and $p^{L}(e)$ are approximately linear with the first derivative of $p^{H}(e)$ only marginally larger than the one of $p^{L}(e)$. Parameters are chosen in a way such that, under transparency, both types of agents choose an effort level that is approximately identical to the maximum possible effort level $E$. If $p^{L}(e)$ and $p^{H}(e)$ are almost identical in this case, both types will be almost indistinguishable for the principal. In addition, a knife-edge case for $B$ is chosen such that, under opacity, it is still beneficial for the high-efficiency type to exert the maximum level of effort $E$. However, for the low-efficiency type this is just not worthwhile. Because $p^{L}(e)$ is almost linear, this implies that $e \approx 0$ is selected by this type. As a consequence, both types behave very differently under opacity.

\subsection{Utility of committee members}

It is also interesting to consider whether committee members would find transparency desirable. A transparency requirement affects committee members' utilities in two ways. First, transparency induces higher effort in the first period, which is detrimental from the members' point of view. Second, it affects the probability of their being re-appointed. It is conceivable that in some cases transparency will increase this probability. However, for large committees one can reach a clear-cut finding:

13 To give another example, we note that $p^{X}(e)=a^{X} e^{\alpha}+b^{X}(X \in\{H, L\})$ satisfies the assumptions laid out in Sect. 2 for $\alpha \in] 0,1\left[, 0<a^{L} \leq a^{H} \leq \min \left\{\frac{1-b^{H}}{E^{\alpha}}, \frac{E^{1-\alpha}}{\alpha \delta B}\right\}\right.$ and $0<b^{L}<b^{H}<1$. It is straightforward but tedious to show that NDQD always holds in this case. 
Proposition 4 For sufficiently large committees, transparency is always detrimental to committee members.

This follows from the fact that for sufficiently large committees the probability of a correct decision being reached and hence of each member being re-appointed converges to one under opacity. This is a consequence of the Condorcet Jury Theorem, which can be applied in our case because of our assumption that $p^{H}(0)>p^{L}(0)>\frac{1}{2}$. This condition implies that each individual committee member is always strictly more likely to choose the correct vote than the wrong vote, even if he exerts no effort. Under transparency, the probability of re-appointment does not depend on committee size and is given by $p^{X}\left(e_{1, T}^{X}\right)$, which is strictly smaller than one. Our finding may explain why many monetary policy committees have adopted transparent decision-making only as a consequence of external pressure. ${ }^{14}$

\section{Unknown own ability}

Up to now, we have assumed that experts know their own ability. However, it is also conceivable that experts do not have superior information about their own efficiency. For example, agents entering a monetary policy committee may not be familiar with the tasks accomplished by this committee and thus may be unsure about their ability regarding these tasks. The case of unknown own ability is considered in this section. ${ }^{15}$

If experts do not know their own abilities, each expert estimates his probability of being highly efficient to be $q^{H}$ and the probability of his being less efficient to be $q^{L}$. It will be useful to introduce

$$
p^{U A}(e):=q^{H} p^{H}(e)+q^{L} p^{L}(e)
$$

which gives the probability of an expert of unknown ability $(U A)$ receiving a correct signal, given that he has chosen effort $e$. Applying the assumptions regarding $p^{L}($.) and $p^{H}($.$) , it is readily verified that p^{U A}(e)$ satisfies

$$
\begin{array}{crrl}
\frac{\partial p^{U A}}{\partial e_{t}^{i}}\left(e_{t}^{i}\right)>0 & \frac{\partial^{2} p^{U A}}{\partial\left(e_{t}^{i}\right)^{2}}\left(e_{t}^{i}\right)<0 & \forall e_{t}^{i} \in[0, E[, \\
\lim _{e_{t}^{i} \rightarrow E} \frac{\partial p^{U A}}{\partial e_{t}^{i}}\left(e_{t}^{i}\right)<\frac{1}{\delta B} & \lim _{e_{t}^{i} \rightarrow 0} \frac{\partial p^{U A}}{\partial e_{t}^{i}}\left(e_{t}^{i}\right)=\infty & \forall e_{t}^{i} \in[0, E[.
\end{array}
$$

\footnotetext{
14 If highly efficient committee members are aiming at attractive future positions, they may advocate transparency, which gives them a platform to improve the public perception of their competence.

15 It will be immaterial to our findings whether committee members learn their ability at the end of the first period or not.
} 
The equilibrium under transparency can be characterized as follows:

Lemma 9 Under transparency, the optimal re-appointment scheme if experts do not know their own ability is

$$
\mu_{T}^{i, U A}\left(Z^{i}\right)= \begin{cases}1 & \text { for } Z^{i}=C \\ 0 & \text { for } Z^{i}=W\end{cases}
$$

All experts vote in line with their private signals and the equilibrium level of effort in the first period is

$$
e_{1, T}^{U A}=\left(\left(p^{U A}\right)^{\prime}\right)^{-1}\left(\frac{1}{\delta B}\right)
$$

The proof is completely analogous to the proofs for transparency and known ability. It is therefore omitted. Similarly, for opacity we obtain

Lemma 10 Under opacity, the optimal re-appointment scheme if experts do not know their own ability is

$$
\mu_{O}^{i, U A}(Z)= \begin{cases}1 & \text { for } Z=C \\ 0 & \text { for } Z=W .\end{cases}
$$

All experts vote in line with their private signals and their equilibrium levels of effort in the first period are

$$
e_{1, O}^{i, U A}=\left(\left(p^{U A}\right)^{\prime}\right)^{-1}\left(\frac{1}{\chi^{i, U A} \delta B}\right)
$$

where $\chi^{i, U A}$ is the probability of member $i$ being pivotal. We note that $\chi^{i, U A}<1$ implies $e_{1, T}^{U A}>e_{1, O}^{i, U A}$. Consequently, $p^{U A}\left(e_{1, T}^{U A}\right)>p^{U A}\left(e_{1, O}^{i, U A}\right) \forall i \in\{1, \ldots, N\}$. Thus the probability of an individual member choosing a correct vote is larger under transparency compared to opacity. In the next proposition we compare the principal's utility under transparency and opacity if experts do not know their own ability.

Proposition 5 With unknown own ability of experts, the principal's first-period utility is always higher under transparency than under opacity, i.e. $u_{1, T}^{P, U A}>u_{1, O}^{P, U A}$.

Proof The probability of a correct decision being reached, $\mathcal{P}\left(p^{1}, p^{2}, \ldots, p^{N}\right)$, is a strictly increasing function of $p^{i} \forall i \in\{1, \ldots, N\}$. This implies

$$
\begin{aligned}
u_{1, T}^{P, U A}=\mathcal{P}\left(p^{U A}\left(e_{1, T}^{U A}\right), p^{U A}\left(e_{1, T}^{U A}\right), \ldots, p^{U A}\left(e_{1, T}^{U A}\right)\right) \\
\quad>\mathcal{P}\left(p^{U A}\left(e_{1, O A}^{1, U A}\right), p^{U A}\left(e_{1, O}^{2, U A}\right), \ldots, p^{U A}\left(e_{1, O}^{N, U A}\right)\right)=u_{1, O}^{P, U A}
\end{aligned}
$$


For experts who know their own ability, we have shown that the principal's secondperiod utility is higher under transparency if NDQD, i.e. (25), holds. For unknown ability of experts, the respective condition is

$$
\frac{d\left(p^{H}\left(e^{U A}(r)\right)-p^{L}\left(e^{U A}(r)\right)\right)}{d r} \geq 0,
$$

where $e^{U A}(r):=\left(\left(p^{U A}\right)^{\prime}\right)^{-1}\left(\frac{1}{r}\right)$. Because of assumption $\frac{\partial p^{H}}{\partial e_{t}^{i}}\left(e_{t}^{i}\right) \geq \frac{\partial p^{L}}{\partial e_{t}^{i}}\left(e_{t}^{i}\right) \forall e_{t}^{i} \in$ $\left[0, E\left[\right.\right.$ and $e^{U A^{\prime}}(r)=-\frac{1}{r^{2} p^{U A^{\prime \prime}}\left(e^{U A}(r)\right)}>0 \forall r \in[0, \delta B]$, Condition (31) always holds. This immediately implies

Proposition 6 With unknown ability, the principal's second-period utility is always higher under transparency than under opacity, i.e. $u_{2, T}^{P, U A}>u_{2, O}^{P, U A}$.

Intuitively, the proposition can be explained in the following way. Under opacity, each expert $i$ chooses an effort level $e_{1, O}^{i, U A}$. Transparency increases this effort level to $e_{1, T}^{U A}$, which makes the probability of a highly efficient expert voting correctly increase more strongly than the respective probability for a less efficient expert. Consequently, the principal can distinguish more easily between highly efficient and less efficient experts, which unambiguously results in higher second-period utility. To sum up, transparency is always beneficial to the principal in both periods if experts do not know their own ability.

\section{Robustness and extensions}

In this section we discuss several issues related to the robustness of our findings and possible extensions to our model. In particular, we focus on the role of the assumption $\left(p^{H}\right)^{\prime}(e) \geq\left(p^{L}\right)^{\prime}(e)$, the possibility of information exchange in pre-meetings, the case where the state of the world cannot be observed by the principal, and the size of committees.

Role of the assumption $\left(p^{H}\right)^{\prime}(e) \geq\left(p^{L}\right)^{\prime}(e)$

It is instructive to discuss the role of the assumption $\left(p^{H}\right)^{\prime}(e) \geq\left(p^{L}\right)^{\prime}(e)$. It ensures that highly efficient members choose higher levels of effort than less efficient ones (see, for example, the first part of Lemma 4), which together with the assumption $p^{H}(e)>p^{L}(e)$ guarantees that highly efficient members are more likely to vote for the correct option than less efficient members. This fact makes it optimal for the principal to re-appoint members who have voted correctly and to dismiss those who have supported the wrong option. One might argue that $\left(p^{H}\right)^{\prime}(e)<\left(p^{L}\right)^{\prime}(e)$ is also plausible at least at some effort levels. For example, it is conceivable that highly efficient members know some easy-to-learn facts without exerting effort. Then, by learning these facts, improving the signal quality would be easier for less efficient members than highly efficient ones.

In the next proposition, we relax the assumption $\left(p^{H}\right)^{\prime}(e) \geq\left(p^{L}\right)^{\prime}(e)$. 
Proposition 7 Suppose we replace assumption $\left(p^{H}\right)^{\prime}(e) \geq\left(p^{L}\right)^{\prime}(e)$ by

$$
p^{H}\left(\left(\left(p^{H}\right)^{\prime}\right)^{-1}(1 / r)\right)>p^{L}\left(\left(\left(p^{L}\right)^{\prime}\right)^{-1}(1 / r)\right) \forall r>0 .
$$

\section{Then all results of Propositions 1 to 4 continue to hold.}

The proof follows from the observation that (32) guarantees that highly efficient members are more likely to choose the correct option in equilibrium in the first period than less efficient members.

In the case where $p^{H}\left(\left(\left(p^{H}\right)^{\prime}\right)^{-1}(1 / r)\right)>p^{L}\left(\left(\left(p^{L}\right)^{\prime}\right)^{-1}(1 / r)\right)$ is violated for some $r$, highly competent members may be less likely to vote correctly than less efficient members when both types choose the effort levels in (13) or (20) respectively. As a consequence, the principal's re-appointment scheme identified in this paper would not be optimal. In Appendix E, we consider such an example. Under transparency, the principal's equilibrium re-appointment scheme corresponds to a mixed strategy, where members who have voted for the wrong option are definitely dismissed and members who have voted for the correct option are re-appointed with positive probability. Both types of members have identical probabilities of voting for the correct option under transparency (otherwise randomizing would not be optimal for the principal). As a result, the principal cannot improve the composition of the committee by her re-appointment decision under transparency. Due to this effect the principal has higher second-period utility under opacity compared to transparency. However, in our example the gains created by transparency in the first period outweigh these losses and the principal receives higher overall utility under transparency.

\section{Pre-meetings}

One interesting extension to our model would incorporate the possibility of members exchanging information in private before the official meeting. ${ }^{16}$ More specifically, suppose that members could exchange messages about their signals and possibly also about their abilities before voting takes place. ${ }^{17}$ Moreover, suppose that this exchange of messages could not be observed by the principal and that all messages were sent simultaneously. Under both transparency and opacity, sending completely uninformative messages would correspond to an equilibrium. Given that all members assume that messages contain no information, it is not profitable for an individual agent to deviate and send a message that is correlated with his signal. For these equilibria, all of our findings would carry over to a scenario with pre-meetings.

However, it is conceivable that additional equilibria exist. Notably under opacity, committee members' interests are aligned as the principal can only observe the outcome of the voting. A higher probability of a correct outcome thus increases the reappointment chances for all members in unison. By exchanging information about their signals, effort levels, and abilities, members may enhance the efficiency of

\footnotetext{
16 Models of voting with prior deliberation have been examined by Austen-Smith and Feddersen (2006), Gerardi and Yariv (2007), Meirowitz (2007), and Swank et al. (2006).

17 We consider pre-meetings in the first period only, as even very small costs incurred by attending a pre-meeting would induce members to abstain from such a meeting in the second period.
} 
information aggregation in the first period, which is beneficial to all of them. This improvement in the efficiency of information aggregation would enable them to prevent being dismissed even for a comparably low aggregate level of their competence. Thus the principal would be less able to improve the composition of the committee by dismissing its members at the beginning of the second period, and hence the probability of a correct decision in the second period would decrease.

Under transparency, there are no incentives to reveal private information truthfully. Given the re-appointment scheme in our paper, revealing private signals and thus improving the precision of information for the other members does not enhance a member's re-appointment chances but only benefits the recipients of the information. In addition, we note that the principal would take the possibility of information exchange into account when deciding whether or not to re-appoint members. Then voting for the wrong option in line with one's colleagues may indicate a particularly low level of competence whereas deviating from the mainstream opinion may reveal an exceptionally high level of competence if that decision turns out to be correct. Hence members may benefit from mistakes of their colleagues, which severely limits the usefulness of pre-vote communication. ${ }^{18}$

To sum up, truthful exchange of information is more likely to represent an equilibrium behavior under opacity. Under opacity, the principal's utility would be higher in the first period but lower in the second period in an equilibrium with truthful exchange of information over and against an equilibrium without informative messages. Additionally, the incentives to exert effort in the first period would remain lower under opacity over and against transparency.

State of the world unobserved by the principal

If the state of the world were unobserved by the principal when she has to re-appoint members, she would not be able to gain any information about members' levels of competence under opacity. Thus re-appointment would be independent of the committee members' effort levels. Then opacity would lead to the lowest possible levels of effort for all committee members and in turn to a low level of utility for the principal in both periods. Under transparency, the principal may attempt to infer information about members' abilities from the pattern of votes. It is plausible that all members would vote in line with their signals. Then it would be optimal for the government to re-appoint those members who share the opinion of the majority but to dismiss the other members because the option chosen by the majority would be more likely to be correct than the other option. In such a scenario, it would be beneficial to show a united front, which might make it attractive to share information in pre-meetings. 19

\footnotetext{
18 See Hahn (2008), who shows in a model of sequential voting without information acquisition that committee members may benefit from wrong decisions of their colleagues. As a consequence, they may not reveal private information truthfully.

19 Visser and Swank (2007) argue that committee members may want to conceal disagreement when all highly efficient members' signals are perfectly correlated. In this case, disagreement signals low levels of ability.
} 
We conclude that, if the state of the world could not be observed by the principal when she makes her re-appointment decisions, opacity would lead to the lowest possible level of welfare. Hence transparency leads to weakly superior results.

\section{Optimal committee size}

Finally we comment on the optimal size of the committee under both transparency regimes. Suppose the principal incurred a certain cost when enlarging the committee, e.g. because she would have to pay a fixed remuneration to experts. Under opacity, an additional member strengthens the other members' free-riding incentives. This effect reduces the gains an additional committee member may imply for the principal. Under transparency, this effect is not present because a member's vote can be observed directly. As a consequence, the committee size that is optimal from the principal's perspective tends to be higher under transparency compared to opacity.

\section{Conclusions}

In this paper we have shown that transparency leads to higher intensity of information collection on the part of committee members, because their individual voting behavior can be observed and they want to appear as competent individuals in order to remain in office. Due to the higher probability of a correct outcome, the principal's first-period utility is always higher under transparency than under opacity. In the second period, the principal's utility is also higher under transparency, unless transparency induces a strong convergence in the signal qualities of highly efficient and less efficient committee members. Then the principal's ability to identify and re-appoint highly efficient members is diminished. If experts do not know their own ability, transparency makes the principal always better off in the second period.

Transparency harms committee members because it forces them to exert more effort. For sufficiently large committees, we have shown that transparency also reduces committee members' re-appointment prospects. This may explain why committee members are sometimes reluctant to publish voting records.

If we take a broader view on whether transparency in committees is desirable, our paper may help to draw a sharper line. If prior investment in knowledge acquisition by committee members is crucial, transparency is advisable. This may be the case in standing expert committees that face heterogeneous and varying tasks. For instance, the council of economic advisers to the Ministry of Economic Affairs in Germany has recently adopted more transparent rules to foster information acquisition. ${ }^{20}$ For other committees, investments into information acquisition are less important, but the abilities of members play an important role. Examples include academic recruiting committees and juries. Then opacity may be justified in light of the literature discussed in the introduction, which identifies distortionary behavioral effects of transparency.

\footnotetext{
20 Source: private communication.
} 
Acknowledgments We would like to thank Johannes Becker, Theresa Fahrenberger, Thomas Lanz, Bernhard Pachl, Bauke Visser, and seminar participants in Berlin and Zurich for valuable comments.

\section{Appendices}

\section{A Proof of Lemma 7}

In the following we analyze under which circumstances it is optimal for the principal to re-appoint or dismiss a particular member $i \in\{1, \ldots, N\}$ under opacity. According to Lemma 2, it is optimal for the principal to re-appoint member $i$ after the first period if the probability of his being highly efficient is strictly higher than $q^{H}$. Conversely it is optimal for the principal to dismiss member $i$ if the probability of his being highly efficient is strictly lower than $q^{H}$.

Now suppose that the decision of the committee has been correct in the first period $(C)$. Then the probability of high efficiency $(X=H)$ of member $i$ is

$$
\begin{aligned}
\operatorname{Pr}(X=H \mid C) & =\frac{\operatorname{Pr}((X=H) \wedge C)}{\operatorname{Pr}(C)} \\
& =\frac{\operatorname{Pr}(C \mid X=H) \operatorname{Pr}(X=H)}{\operatorname{Pr}(C)} \\
& =\frac{\operatorname{Pr}(C \mid X=H)}{\operatorname{Pr}(C)} \cdot q^{H} \\
& =\frac{\operatorname{Pr}(C \mid X=H)}{q^{H} \operatorname{Pr}(C \mid X=H)+\left(1-q^{H}\right) \operatorname{Pr}(C \mid X=L)} \cdot q^{H} \\
& =\frac{1}{q^{H}+\left(1-q^{H}\right) \frac{\operatorname{Pr}(C \mid X=L)}{\operatorname{Pr}(C \mid X=H)}} \cdot q^{H} \\
& >q^{H} .
\end{aligned}
$$

Here we have used $\operatorname{Pr}(C \mid X=L)<\operatorname{Pr}(C \mid X=H)$, which follows from the fact that member $i$ is pivotal with strictly positive probability in the first period and that a highly efficient member is always strictly more likely to vote correctly than a less efficient member (see Lemma 6). As a consequence, it is optimal to re-appoint member $i$ if the decision in the first period has been correct.

Analogously one can show $\operatorname{Pr}(X=H \mid W)<q^{H}$, where $W$ denotes a wrong decision in the first period. This implies that it is optimal for the principal to dismiss member $i$ in this case.

\section{B Proof of Lemma 8}

Consider the hypothetical $2 N$-player normal-form game with players $(i, X) \in$ $\{1, \ldots, N\} \times\{H, L\}$, strategies $e_{1}^{i, X} \in[0, E]$, and payoff functions $\phi^{i, X}$. The second derivative of the payoff function amounts to 


$$
\frac{\partial^{2} \phi^{i, X}}{\partial\left(e_{1}^{i, X}\right)^{2}}=\delta \chi^{i} \cdot\left(p^{X}\right)^{\prime \prime}\left(e_{1}^{i, X}\right)\left[\mu_{O}^{i}(C)-\mu_{O}^{i}(W)\right] B<0 .
$$

Hence $\phi^{i, X}$ is a strictly concave function of $e_{1}^{i, X}$. Moreover, $\phi^{i, X}$ is a continuous function of the other players' effort choices, because $\chi^{i}$ is a continuous function of the other players' effort choices. ${ }^{21}$ Thus the hypothetical game has an equilibrium in pure strategies (see Theorem 1.2 in Fudenberg and Tirole (1991, p. 34); the theorem goes back to Debreu (1952), Fan (1952), and Glicksberg (1952)).

\section{Proof of Proposition 2}

\section{C.1 Step 1}

We divide the proof into three steps. In the first step we will introduce some notation that will be useful for the remainder of the analysis. Now we deal with the probability of a correct outcome in the second period as a function of the number of highly efficient members. Recall that all members exert no effort in the second period. Then this probability can be written as

$$
\pi_{n}=\mathcal{P}(\underbrace{p^{H}(0), \ldots, p^{H}(0)}_{n \text { times }}, \underbrace{p^{L}(0), \ldots, p^{L}(0)}_{N-n \text { times }}) .
$$

Because $\mathcal{P}\left(p^{1}, \ldots, p^{N}\right)$ is strictly increasing in all of its arguments, it is obvious that $\pi_{n}$ is a strictly increasing function of $n$. Intuitively, the more highly efficient members there are, the higher is the probability of a correct decision in the second period, because highly efficient members vote correctly with probability $p^{H}(0)$, whereas less efficient members only make a correct decision with probability $p^{L}(0)<p^{H}(0)$.

For given numbers of highly efficient and less efficient members the principal's utility in the second period is independent of the transparency regime. However, the distribution of highly efficient and less efficient members will be different under transparency and opacity. Therefore it is necessary to analyze this distribution under both scenarios. We use $\xi_{R, n}$ to denote the probability of $n$ members being highly efficient in the second period under regime $R \in\{T, O\}$.

We derive $\xi_{T, n}$ first. In the second period, the probability of an individual expert being highly efficient under transparency is

$$
Q_{2, T}^{H}:=q^{H} p^{H}\left(e_{1, T}^{H}\right)+\left\{q^{H}\left[1-p^{H}\left(e_{1, T}^{H}\right)\right]+\left(1-q^{H}\right)\left[1-p^{L}\left(e_{1, T}^{L}\right)\right]\right\} q^{H} .
$$

The first summand gives the probability of the member in the first period being highly efficient and choosing the correct vote, which entails re-appointment. The second

\footnotetext{
21 Note that $\chi^{i}$ is a continuous function of the probabilities of individual members choosing a particular vote, which in turn are continuous functions of the other players' effort choices.
} 
summand corresponds to the eventuality that an expert may choose a wrong vote and be replaced by a highly efficient expert. Using $Q_{2, T}^{H}$, we can write $\xi_{T, n}$ as

$$
\xi_{T, n}=\left(\begin{array}{c}
N \\
n
\end{array}\right)\left(Q_{2, T}^{H}\right)^{n}\left(1-Q_{2, T}^{H}\right)^{N-n} .
$$

The expression for the respective probability under opacity, $\xi_{O, n}$, is much more complex, because the probability of an individual committee member being highly efficient depends on the outcome of the voting and thus on the behavior of all committee members rather than the individual's behavior alone. Thus we refrain from stating it explicitly.

The principal's expected utility in the second period under regime $R \in\{T, O\}$ can be expressed as

$$
u_{2, R}^{P}=\sum_{n=0}^{N} \xi_{R, n} \pi_{n}
$$

The claim of the proposition can be written as

$$
\sum_{n=0}^{N} \xi_{T, n} \pi_{n}=u_{2, T}^{P}>u_{2, O}^{P}=\sum_{n=0}^{N} \xi_{O, n} \pi_{n} .
$$

Now we introduce the hypothetical distribution one would obtain under transparency in the second period if all members chose the effort levels optimal under opacity $\left(e_{1, O}^{i, X}\right)$ in the first period. The respective probability mass function will be denoted by $\widetilde{\xi}_{T, n}$.

In step 2 we will show $\sum_{n=0}^{N} \xi_{T, n} \pi_{n} \geq \sum_{n=0}^{N} \widetilde{\xi}_{T, n} \pi_{n}$. In step 3 we will derive $\sum_{n=0}^{N} \widetilde{\xi}_{T, n} \pi_{n}>\sum_{n=0}^{N} \xi_{O, n} \pi_{n}$. Combining steps 2 and 3 delivers (37).

\section{2 Step 2}

In this step we show $\sum_{n=0}^{N} \xi_{T, n} \pi_{n} \geq \sum_{n=0}^{N} \widetilde{\xi}_{T, n} \pi_{n}$. We have already noted that $\pi_{n}$ is strictly increasing in $n$. Thus it is sufficient to show that the distribution with the probability mass function $\xi_{T, n}$ weakly first-order stochastically dominates the one that is characterized by $\widetilde{\xi}_{T, n}$.

We now define $\widetilde{Q}_{2, T}^{i, H}$ as

$$
\widetilde{Q}_{2, T}^{i, H}:=q^{H} p^{H}\left(e_{1, O}^{i, H}\right)+\left\{q^{H}\left[1-p^{H}\left(e_{1, O}^{i, H}\right)\right]+\left(1-q^{H}\right)\left[1-p^{L}\left(e_{1, O}^{i, L}\right)\right]\right\} q^{H} .
$$

This expression is identical to $Q_{2, T}^{H}$ as defined in Equation (34) except for the fact that we have replaced the effort levels under transparency by the effort levels under 
opacity. Thus $\widetilde{Q}_{2, T}^{i, H}$ corresponds to the probability under transparency of an individual member being highly efficient in the second period assuming that all members have chosen the effort levels they would find optimal under opacity in the first period and that the principal applies the re-appointment scheme specified in Lemma 5.

Recall that $\xi_{T, n}$ is the probability of $n$ members being highly efficient if the probability of an individual member being highly efficient is probability $Q_{2, T}^{H}$. Similarly, $\widetilde{\xi}_{T, n}$ is the probability of $n$ members being highly efficient if the probability of each member $i$ being highly efficient amounts to $\widetilde{Q}_{2, T}^{i, H}$.

Consequently, the distribution characterized by the probability mass function $\xi_{T, n}$ weakly first-order stochastically dominates the distribution described by $\widetilde{\xi}_{T, n}$ if $Q_{2, T}^{H} \geq \widetilde{Q}_{2, T}^{i, H} \forall i \in\{1, \ldots, N\}$, which means that the individual probability of being highly efficient under transparency in the second period is higher for each member if all members choose the effort levels optimal under transparency rather than those optimal under opacity. This is shown in the following. Using (34) and (38), we obtain

$$
\begin{aligned}
Q_{2, T}^{H}-\widetilde{Q}_{2, T}^{i, H}= & q^{H}\left(p^{H}\left(e_{1, T}^{H}\right)-p^{H}\left(e_{1, O}^{i, H}\right)\right) \\
& -\left\{q^{H}\left[p^{H}\left(e_{1, T}^{H}\right)-p^{H}\left(e_{1, O}^{i, H}\right)\right]+\left(1-q^{H}\right)\left[p^{L}\left(e_{1, T}^{L}\right)-p^{L}\left(e_{1, O}^{i, L}\right)\right]\right\} q^{H} \\
= & q^{H}\left(1-q^{H}\right)\left[p^{H}\left(e_{1, T}^{H}\right)-p^{H}\left(e_{1, O}^{i, H}\right)-\left(p^{L}\left(e_{1, T}^{L}\right)-p^{L}\left(e_{1, O}^{i, L}\right)\right)\right] .
\end{aligned}
$$

This expression is weakly positive if

$$
p^{H}\left(e_{1, T}^{H}\right)-p^{L}\left(e_{1, T}^{L}\right)-\left(p^{H}\left(e_{1, O}^{i, H}\right)-p^{L}\left(e_{1, O}^{i, L}\right)\right) \geq 0
$$

This condition is fulfilled if NDQD holds. Thus $\sum_{n=0}^{N} \xi_{T, n} \pi_{n} \geq \sum_{n=0}^{N} \widetilde{\xi}_{T, n} \pi_{n}$ holds.

\section{C.3 Step 3}

Finally, we show $\sum_{n=0}^{N} \widetilde{\xi}_{T, n} \pi_{n}>\sum_{n=0}^{N} \xi_{O, n} \pi_{n}$. Let us consider the transparency scenario but assume that all members choose the effort levels that correspond to those under opacity. It is straightforward to see that Lemma 5 can also be applied to the case where members choose $e_{1, O}^{i, X}$ rather than $e_{1, T}^{X}$. Hence it is strictly optimal for the principal to re-appoint a member who has chosen the correct decision and to dismiss a member who has voted for the wrong decision. In particular, this behavior is strictly preferred to the behavior where the principal re-appoints all members if and only if the overall decision has been correct. Thus $\sum_{n=0}^{N} \widetilde{\xi}_{T, n} \pi_{n}$ represents the maximum level of welfare and $\sum_{n=0}^{N} \widetilde{\xi}_{T, n} \pi_{n}>\sum_{n=0}^{N} \xi_{O, n} \pi_{n}$.

We have already noted that steps 2 and 3 jointly guarantee the proposition. 


\section{Example where NDQD is violated}

In the following we construct an example where NDQD is violated and opacity leads to a higher utility of the principal in the second period. Suppose $N=3, E=1$, $q_{H}=1 / 2$, and

$$
\begin{aligned}
p^{H}(e) & =\frac{1}{2}+\underline{\varepsilon}+\left(\frac{1}{2}-\underline{\varepsilon}\right) e, \\
p^{L}(e) & =\frac{1}{2}+\left(\frac{1}{2}-\bar{\varepsilon}\right) e
\end{aligned}
$$

where $\bar{\varepsilon}>\underline{\varepsilon}$. It is clear that these functions do not satisfy two of the constraints introduced before, as they are not strictly concave and $\lim _{e_{t}^{i} \rightarrow 0} \frac{\partial p^{X}}{\partial e_{t}^{i}}\left(e_{t}^{i}\right)=\infty \forall X \in\{H, L\}$ does not hold. ${ }^{22}$ However, it is always possible to find functions that are arbitrarily close to $p^{H}(e)$ and $p^{L}(e)$ and satisfy these assumptions.

Let us use $\tilde{\chi}$ to denote the probability of an individual member being pivotal, conditional on highly efficient colleagues voting for the correct option with probability one and less efficient ones with probability one half. Now suppose that $B$ is the unique solution to

$$
\begin{aligned}
\frac{1}{\tilde{\chi} \delta B} & =\frac{1}{2}\left(\left(p^{L}\right)^{\prime}(e)+\left(p^{H}\right)^{\prime}(e)\right) \\
& =\frac{1}{2}-\frac{1}{2}(\underline{\varepsilon}+\bar{\varepsilon})
\end{aligned}
$$

Hence $\left(p^{L}\right)^{\prime}(e)=(1 / 2-\bar{\varepsilon})<1 /(\widetilde{\chi} \delta B)<(1 / 2-\underline{\varepsilon})=\left(p^{H}\right)^{\prime}(e)$. This condition implies that under opacity highly efficient members choose the maximum effort of one, whereas less efficient members choose no effort at all. Under transparency, both types choose the maximum effort; this follows from $\widetilde{\chi}<1$. Moreover, suppose that $\underline{\varepsilon}$ and $\bar{\varepsilon}$ with $(\underline{\varepsilon}<\bar{\varepsilon})$ are extremely small such that $p^{L}(1) \approx p^{H}(1)=1$. Importantly, both types of members have approximately the same probability of choosing the correct option under transparency in the first period.

It is plausible (and can also be verified formally) ${ }^{23}$ that for this particular constellation the principal cannot improve the composition of the committee under transparency by selecting individual members because both types have probabilities of voting correctly that are arbitrarily close to one another. By contrast, under opacity moderate improvements of the committee composition are feasible, which lead to a somewhat higher probability of a correct decision in the second period over and against transparency.

\footnotetext{
22 In a similar vein, it is not clear whether $\lim _{e_{t}^{i} \rightarrow 1} \frac{\partial p^{X}}{\partial e_{t}^{i}}\left(e_{t}^{i}\right)<1 /(\delta B) \forall X \in\{H, L\}$. Moreover, $p^{L}(0)>$ $1 / 2$ does not hold strictly.

23 A proof is available upon request.
} 


\section{E Example where $p^{\mathrm{H}}\left(\left(\left(p^{\mathrm{H}}\right)^{\prime}\right)^{-1}(1 / \mathbf{r})\right)>\mathbf{p}^{\mathrm{L}}\left(\left(\left(\mathbf{p}^{\mathrm{L}}\right)^{\prime}\right)^{-1}(1 / \mathbf{r})\right)$ is violated}

Suppose $q_{H}=1 / 2, E=1, \delta=1, B=4$, and

$$
\begin{aligned}
& p^{H}(e)=\frac{3}{4}+\frac{1}{4} \sqrt{e}, \\
& p^{L}(e)=\frac{1}{2}+\frac{1}{2} \sqrt{e} .
\end{aligned}
$$

Then the first derivatives of $p^{H}(e)$ and $p^{L}(e)$ are

$$
\begin{aligned}
& \left(p^{H}\right)^{\prime}(e)=\frac{1}{8} \frac{1}{\sqrt{e}}, \\
& \left(p^{L}\right)^{\prime}(e)=\frac{1}{4} \frac{1}{\sqrt{e}} .
\end{aligned}
$$

Consequently, $p^{H}(e)>p^{L}(e) \forall e<1$ and $\left(p^{H}\right)^{\prime}(e)<\left(p^{L}\right)^{\prime}(e) \forall e \leq 1$. Suppose, for the moment, that the principal rewarded correct decisions with a payment of $r$. Then the optimal effort levels $e^{H}$ and $e^{L}$ can be computed as follows:

$$
\begin{aligned}
& \frac{1}{r}=\left(p^{H}\right)^{\prime}(e)=\frac{1}{8} \frac{1}{\sqrt{e^{H}}}, \\
& \frac{1}{r}=\left(p^{L}\right)^{\prime}(e)=\frac{1}{4} \frac{1}{\sqrt{e^{L}}}
\end{aligned}
$$

Solving for $e^{H}$ and $e^{L}$ yields $e^{H}=(r / 8)^{2}$ and $e^{L}=(r / 4)^{2}$. Inserting these values into $p^{H}(e)$ and $p^{L}(e)$, we obtain $p^{H}\left(e^{H}\right)=3 / 4+(1 / 4) \cdot(r / 8)^{2}$ and $p^{L}\left(e^{L}\right)=1 / 2+(1 / 2) \cdot(r / 4)^{2}$. Under the assumption that the principal chooses the re-appointment scheme from Lemma 5 under transparency, the optimal behavior of agents can be computed by inserting $r=4$ into these equations, which gives $p^{H}\left(e^{H}\right)=7 / 8$ and $p^{L}\left(e^{L}\right)=1$. Consequently, $p^{L}\left(e^{L}\right)>p^{H}\left(e^{H}\right)$, or, equivalently $p^{H}\left(\left(\left(p^{H}\right)^{\prime}\right)^{-1}(1 / r)\right)<p^{L}\left(\left(p^{L}\right)^{\prime}(1 / r)\right)$ for $r=4$. This implies that the re-appointment scheme from Lemma 5 is not optimal, as the principal would prefer to dismiss members who have chosen the correct option and re-appoint the other members.

Now let us consider the following candidate equilibrium under transparency: $\widetilde{e}^{H}=$ $1 / 9, \widetilde{e}^{L}=4 / 9, \mu(C)=2 / 3$, and $\mu(W)=0$. By setting $r=\delta B(\mu(C)-\mu(W))=$ $8 / 3$, it is straightforward to demonstrate that $\left(p^{L}\right)^{\prime}\left(\widetilde{e}^{L}\right)=\left(p^{H}\right)^{\prime}\left(\widetilde{e}^{H}\right)=3 / 8=1 / r$ holds, which implies that both types of members cannot profitably deviate. In addition, randomizing is optimal for the principal because $p^{L}\left(\widetilde{e}^{L}\right)=p^{H}\left(\widetilde{e}^{H}\right)=5 / 6$. Hence the proposed equilibrium actually exists under transparency.

Under opacity, the equilibrium considered in the paper exists, as can be derived easily. ${ }^{24}$ The effort levels are $\hat{e}^{H} \approx 0.145$ and $\hat{e}^{L} \approx 0.036$, which implies $p^{H}\left(\hat{e}^{H}\right) \approx$ $0.798, p^{L}\left(\hat{e}^{L}\right) \approx 0.690$, and $\chi \approx 0.381$.

\footnotetext{
24 Detailed computations are available upon request.
} 
Hence the probability of choosing the correct option is higher for both types under transparency over and against opacity $\left(p^{L}\left(\widetilde{e}^{L}\right)=5 / 6>p^{H}\left(\hat{e}^{H}\right) \approx 0.798\right.$ and $\left.p^{L}\left(\widetilde{e}^{L}\right)=5 / 6>p^{L}\left(\hat{e}^{L}\right) \approx 0.690\right)$. As a result, the principal benefits from transparency in the first period. Because both types have identical probabilities of choosing the correct option in the first period under transparency, it is impossible to improve the committee by re-appointing or dismissing members. By contrast, moderate improvements of the committee composition are feasible under opacity, where highly efficient members have a strictly higher probability of voting for the correct option in the first period than their less efficient colleagues. This implies that the principal is harmed by transparency in the second period. It can be shown that the gains from opacity in the second period do not outweigh the losses in the first period. Hence the principal prefers transparency.

\section{References}

Austen-Smith D, Feddersen T (2006) Deliberation, preference uncertainty and voting rules. Am Polit Sci Rev 100:209-218

Debreu G (1952) A social equilibrium existence theorem. Proc Natl Acad Sci USA 38(10):886-893

Fan K (1952) Fixed-point and minimax theorems in locally convex topological linear spaces. Proc Natl Acad Sci USA 38(2):121-126

Fingleton J, Raith M (2005) Career concerns of bargainers. J Law Econ Organ 21(1):179-204

Fudenberg D, Tirole J (1991) Game theory. MIT Press, Cambridge, MA

Gerardi D, Yariv L (2007) Deliberative voting. J Econ Theory 134(1):317-338

Gerardi D, Yariv L (2008) Information acquisition in committees. Game Econ Behav 62(2):436-459

Gerling K, Grüner HP, Kiel A, Schulte E (2005) Information acquisition and decision making in committees: a survey. Eur J Polit Econ 21(3):563-597

Gersbach H (1995) Information efficiency and majority decisions. Soc Choice Welfare 12(4):363-370

Gersbach H, Hahn V (2008) Should the individual voting records of central-bank council members be published? Soc Choice Welfare 30(4):655-683

Glicksberg IL (1952) A further generalization of the Kakutani fixed point theorem, with application to Nash equilibrium points. Proc Am Math Soc 3(1):170-174

Hahn V (2008) Committees, sequential voting and transparency. Math Soc Sci 63(3):366-385

Holmström B (1982) Managerial incentive problems: a dynamic perspective. In essays in economics and management in honor of Lars Wahlbeck. Swedish School of Economics, Helsinki, 1982. Reprinted in: 1999. Rev Econ Stud 66(1):169-182

Levy G (2007) Decision making in committees: transparency, reputation and voting rules. Am Econ Rev 97(1):150-168

Marschak J, Radner R (1972) Economic theory of teams. Yale University Press, New Haven and London. Cowles Foundation for Research in Economics at Yale University, Monograph 22

Meirowitz A (2007) In defense of exclusionary deliberation: communication and voting with private beliefs and values. J Theor Polit 19(3):301-327

Mukhopadhaya K (2003) Jury size and the free rider problem. J Law Econ Organ 19(1):24-44

Ottaviani M, Sørensen PN (2001) Information aggregation in debate: who should speak first? J Public Econ 81(3):393-421

Persico N (2004) Committee design with endogenous information. Rev Econ Stud 71(246):165-191

Prat A (2005) The wrong kind of transparency. Am Econ Rev 95(3):862-877

Scharfstein D, Stein J (1990) Herd behavior and investment. Am Econ Rev 80(3):465-479

Sibert A (2003) Monetary policy committees: individual and collective reputation. Rev Econ Stud 70(3):649-665

Stasavage D (2007) Polarization and publicity: rethinking the benefits of deliberative democracy. J Polit 69(1):59-72 
Swank J, Swank O, Visser B (2006) Transparency and pre-meetings. Tinbergen Institute Discussion Papers 06-051/1, Tinbergen Institute

Trueman B (1994) Analyst forecasts and herding behavior. Rev Financ Stud 7(1):97-124

Visser B, Swank O (2007) On committees of experts. Q J Econ 122(1):337-372 